\section{Field-portable Analysis of Anthocyanin Concentration in Sepals of Hydrangea macrophylla}

\author{
Henry D. Schreiber ${ }^{1}$ and Nicholas A. Wade \\ Department of Chemistry, Virginia Military Institute, Lexington, VA 24450
}

Additional index words. pigment, sepal color, chlorophyll meter, bigleaf hydrangea, aluminum

\begin{abstract}
A field-portable method, adapting commercially available chlorophyll content meters, has been developed to measure the anthocyanin concentration in red and blue sepals of Hydrangea macrophylla. The meters were calibrated to total extractable anthocyanin concentration. The strength of the linear relationship (anthocyanin content index vs. extractable anthocyanin concentration) was better for red than blue sepals, due perhaps to the inclusion of purplish sepals in the blue data set or perhaps to more than one mechanism for the bluing of sepals. The meters were demonstrated to be an effective tool for the measurement of relative anthocyanin concentration in hydrangea sepals as a function of bloom stage.
\end{abstract}

Many cultivars of Hydrangea macrophylla produce inflorescences bearing sepal colors that reflect the soil $\mathrm{pH}$; such sepals are pink-red when the shrub is grown in neutral to basic soils and blue when grown in acid soils. The primary pigment in the sepals is an anthocyanin, delphinidin-o-glucoside (Takeda et al., 1985), whose flavylium cation provides the characteristic pink-red color to hydrangea sepals (Moncada et al., 2003). Sepals turn blue because this anthocyanin forms a complex with aluminum as $\mathrm{Al}^{3+}$ (Toyama-Kato et al., 2003), with the coloration intensified and stabilized by other copigments (Kondo et al., 2005). Accordingly, because $\mathrm{Al}^{3+}$ is mobile only in acidic soils, the bluing of the hydrangea sepals is thought to follow the availability of $\mathrm{Al}^{3+}$ to the shrub.

Prior studies (Asen et al., 1960; Robinson, 1939) provided evidence that the concentration of delphinidin- $o$-glucoside in blue sepals differed from that in red sepals, consequently contributing to the color change in hydrangea inflorescences. Although the role of $\mathrm{Al}^{3+}$ in the bluing of the sepals has been firmly ascertained (Kondo et al., 2005), it is still not certain whether this is the sole mechanism for the bluing (Pharr et al., 2006). Thus, to establish a comprehensive chemical basis for the unique color change in hydrangea sepals, the anthocyanin concentrations in sepals need to be determined as a function of cultivar, color, and growing conditions. Such studies may lead to the development of alternative mechanisms for coloration and perhaps to methods for the bluing of sepals when hydrangeas are grown in neutral or

Received for publication 2 Mar. 2007. Accepted for publication 29 Apr. 2007.

This work was supported by grants from the Thomas F. \& Kate Miller Jeffress Memorial Trust and the Virginia Military Institute.

${ }^{1}$ To whom reprint requests should be addressed; e-mailHS@vmi.edu basic soils or for generating novel sepal colors, such as yellow or orange.

Furthermore, there is a need for rapid field-portable measurements of anthocyanin content in hydrangea sepals, as determinations of extractable anthocyanin concentration are typically lengthy and destroy the inflorescence. Such measurements will allow the simultaneous analyses of other components (for example, $\mathrm{Al}^{3+}$ ) in the same sepals. The method would also allow quick and quantitative measurements of the effect of such variables as climate, bloom stage, fertilizer, chemical treatments, and sunlight on the anthocyanin concentration and, thus, the color intensity of the sepals.

\section{Materials and Methods}

Hydrangea cultivars. Cultivars were purchased or obtained from Hydrangeas Plus (VanHoose Enterprises, Aurora, OR), Wayside Gardens (Hodges, SC), and the Center for Applied Nursery Research (Dearing, GA). The following cultivars were used in this study: H. macrophylla var. macrophylla (hortensia or mophead) 'All Summer Beauty', 'Domotoi', 'Dooley', 'Endless Summer', 'Enziandom', 'Generale Vicomtesse DeVibraye', 'Hamburg', 'Marechal Foch', 'Masja', 'Nikko Blue', and 'Penny Mac'; and H. macrophylla var. normalis (lacecap) 'Eisvogel' and 'Kardinal'. Some of the inflorescences were harvested from stock planted in pots containing a commercially available nursery-blend tree and shrub medium. Other inflorescences were harvested from mature hydrangea shrubs grown at BackCountry Research (Rockbridge County, VA). Bloom colors from both sources were adjusted by chemical treatments, such as lime, aluminum sulfate, ferric sulfate, ammonium molybdate, and citric acid.

Extractable anthocyanin concentration. A method was developed to quantitatively determine the extractable anthocyanin con- centration of hydrangea sepals. Details of the procedure have been previously reported (Pharr et al., 2006) and were based on a standard method of differential spectrophotometry (Giusti and Wrolstad, 2000). In essence, the anthocyanin was first extracted into an acidified methanol solution. Peak and baseline absorbances of aliquots of this extract in two different $\mathrm{pH}$ buffers were then measured. The resulting differential absorbance was finally related to anthocyanin concentration, expressed as mg of delphinidin-o-glucoside per $g$ of fresh sepal.

Analyses yielded extractable anthocyanin concentrations ranging from 0.06 to $0.48 \mathrm{mg}$ of anthocyanin per $\mathrm{g}$ of fresh hydrangea sepal for the cultivars studied. There have been only a few other reports of quantitative analyses of the anthocyanin concentration of hydrangea sepals. For example, 'Merveille' was reported to have $2.6-3.0 \mathrm{mg}$ of anthocyanin per $\mathrm{g}$ of dry sepal (Asen et al., 1957). To afford comparison, this study determined that an average hydrangea sepal lost $87.5 \%( \pm 1.9 \%)$ of its mass upon drying, converting this previous determination to an equivalent measurement of $\approx 0.35 \mathrm{mg}$ of anthocyanin per $g$ of fresh sepal, which is in general agreement with this study.

Field-portable anthocyanin concentration. Studies have demonstrated that the portable CCM-200 Chlorophyll Content Meter (OptiSciences, Tyngsboro, MA) accurately measures the relative chlorophyll content in leaves (van den Berg and Perkins, 2004). The CCM calculates a chlorophyll content index (CCI) from the ratio of optical absorbance at $655 \mathrm{~nm}$ to that at $940 \mathrm{~nm}$. Another study has also shown that this CCM can be adapted to measure anthocyanin concentration (redness) in autumn sugar maple leaves by replacing the $655 \mathrm{~nm}$ light-emitting diode (LED) with a 530-nm LED (van den Berg and Perkins, 2005). Such a replacement is commercially available from the manufacturer so that the instrument measures absorbance at the wavelength appropriate to the principal anthocyanin in the red leaves. As red hydrangea sepals display maximum absorbance at $\approx 540 \mathrm{~nm}$ and blue sepals at $\approx 590 \mathrm{~nm}$ (Yoshida et al., 2003), the manufacturer supplied two different meters adapted from the CCM-200, one whose measuring LED was $540 \mathrm{~nm}$ to estimate the relative red anthocyanin content index (RACI), and the other with a 590-nm LED to measure the blue anthocyanin content index (BACI). Furthermore, the measuring probes of both meters were decreased to $1 / 4$-inch in diameter.

Before sepals in a harvested inflorescence were analyzed for extractable anthocyanin concentration, at least 20 of its sepals were measured with the appropriate anthocyanincontent meter. The same sepals measured for RACI or BACI were then used in extractable anthocyanin determinations. White sepals from other cultivars were also measured to define zero points for both the red- and bluemonitoring meters. A standard micrometer was used to determine the thicknesses of the harvested sepals. 
The portable anthocyanin content meters were used in the field to measure the RACI or BACI of red and blue sepals, respectively. Measurements were made as a function of bloom stage to illustrate the potential applications of the instrument. Once again, at least 20 sepals (one meter reading for each sepal) were measured per inflorescence, and the samples were measured only when sufficient sepals were large enough (that is, $>1 / 4$ inch) to use the probe.

\section{Results and Discussion}

Data points for the RACI vs. extractable anthocyanin concentration for red sepals collected from multiple hydrangea cultivars indicate a linear relationship, suggesting that the meter effectively estimates the anthocyanin concentration of the sepals (Fig. 1). The strength of the linear relationship $\left(r^{2}\right)$ was about the same as that reported for autumn maple leaves (van den Berg and Perkins, 2005). One requirement of this method is the need for a large sampling of hydrangea sepals from a single inflorescence to obtain an average RACI, as there was $\approx 10 \%-30 \%$ relative standard deviation in meter readings. This is representative of the variation in the color intensities of the individual sepals, not of the limitations in the meter. Whereas an inflorescence appeared to be a homogeneous color, sepal-to-sepal variation was visually obvious.

The strength of the linear relationship to extractable anthocyanin concentration was poorer for the BACI of the blue sepals (Fig. 2) than for the RACI of the red sepals (Fig. 1). The highest concentration samples (those of 'Kardinal') were not included in the linear regression analysis of Fig. 2 due to concerns over meter reading saturation for these extremely dark samples. Although attempts were made to select only "true" blue samples, purple-tinted sepals were more likely to contaminate the sample pool for the blue sepals than for the red sepals. Such purplish sepals would have a wavelength of maximum absorbance less than the 590-nm LED used to monitor the blue sepals. As such, one limitation for this method is that it is appropriate only for red or blue sepals; purple sepals absorb at a continuum of wavelengths between those two extremes, making it difficult to design a meter accordingly. Alternatively, the scatter in the Fig. 2 data may be evidence that there are multiple mechanisms to form blue chromophores, each with its own molar absorptivity at a somewhat different wavelength, in the hydrangea sepals.

The spectral profiles of the colored sepals were sufficiently broad so that the red sepals would also register a small value for BACI and the blue sepals a small value for RACI. Thus, the RACI-adapted meter was only used for red sepals, and the BACI-adapted meter only for blue sepals. Other measurement functions, such as RACI - BACI and BACI RACI for the red and blue sepals, respectively, did not afford better calibrations to the extractable anthocyanin concentrations.
Beer's law states that the absorbance divided by the path length is proportional to the concentration of the absorbing species. Thus, the meter reading (RACI or BACI) divided by sepal thickness might be expected to provide an even better calibration to the extractable anthocyanin concentration of the sepals. However, there was no improvement in the strength of the linear calibration by plotting RACI/thickness or BACI/thickness as a function of the extractable anthocyanin concentration. This result is confirmation that the meters were designed to eliminate the influence on tissue thickness on the index readings. Interestingly, the extractable anthocyanin concentration tended to increase with

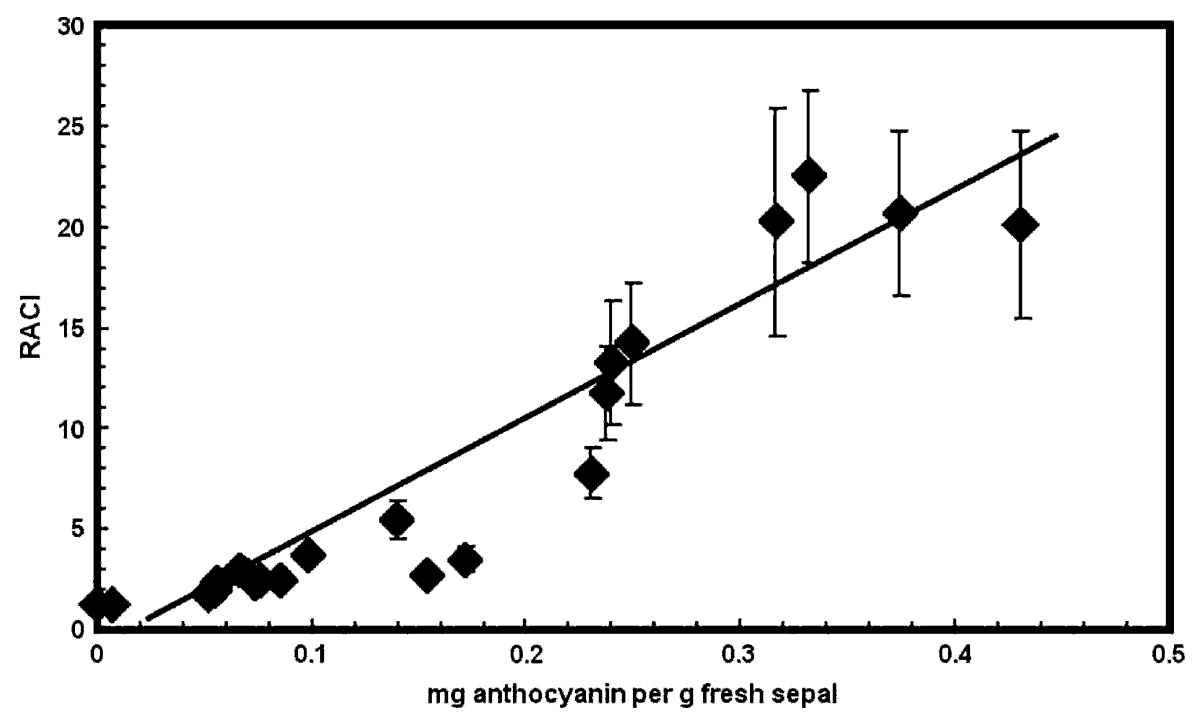

Fig. 1. Red anthocyanin content index (RACI) as a function of extractable anthocyanin concentration (mg of delphinidin-o-glucoside per g of fresh sepal) for the red sepals of multiple hydrangea cultivars. Linear regression resulted in the equation $y=56.7 x-1.5\left(r^{2}=0.87\right)$.

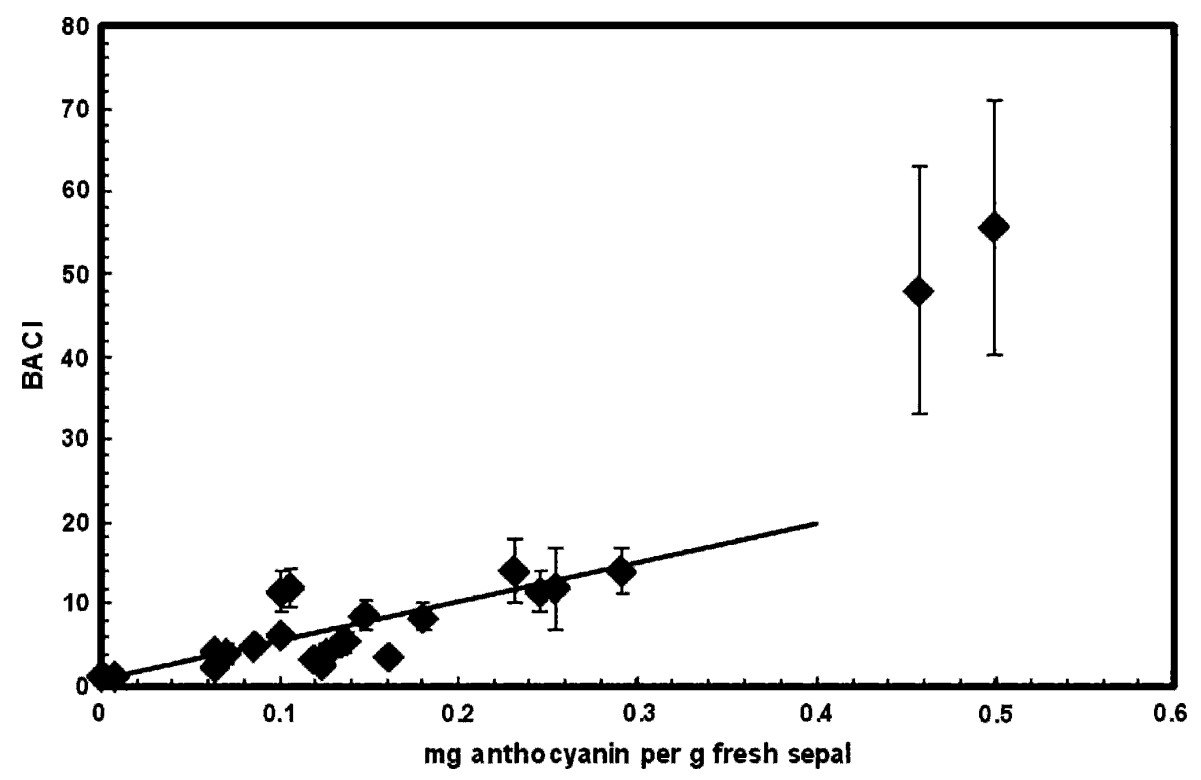

Fig. 2. Blue anthocyanin content index (BACI) as a function of extractable anthocyanin concentration (mg of delphinidin-o-glucoside per $\mathrm{g}$ of fresh sepal) for the blue sepals of multiple hydrangea cultivars. Linear regression, exclusive of the two data points of highest concentration, resulted in the equation $y=42.7 x+1.1\left(r^{2}=0.60\right)$. sepal thickness (Fig. 3). Nevertheless, the variation in the calibration plot is not due to dilution of the pigment layer by a larger percentage of sepal mass (as monitored by thickness) but to the pigment layer increasing proportion to the sepal thickness. were used to follow the color of several hydrangeas' inflorescences over the course of a bloom cycle. One such tracking is shown in Fig. 4 for a red 'Masja' inflorescence, nin content with time. Zero time was defined to be when sufficient sepals reached a size of $1 / 4$ inch so that measurements could be taken; 


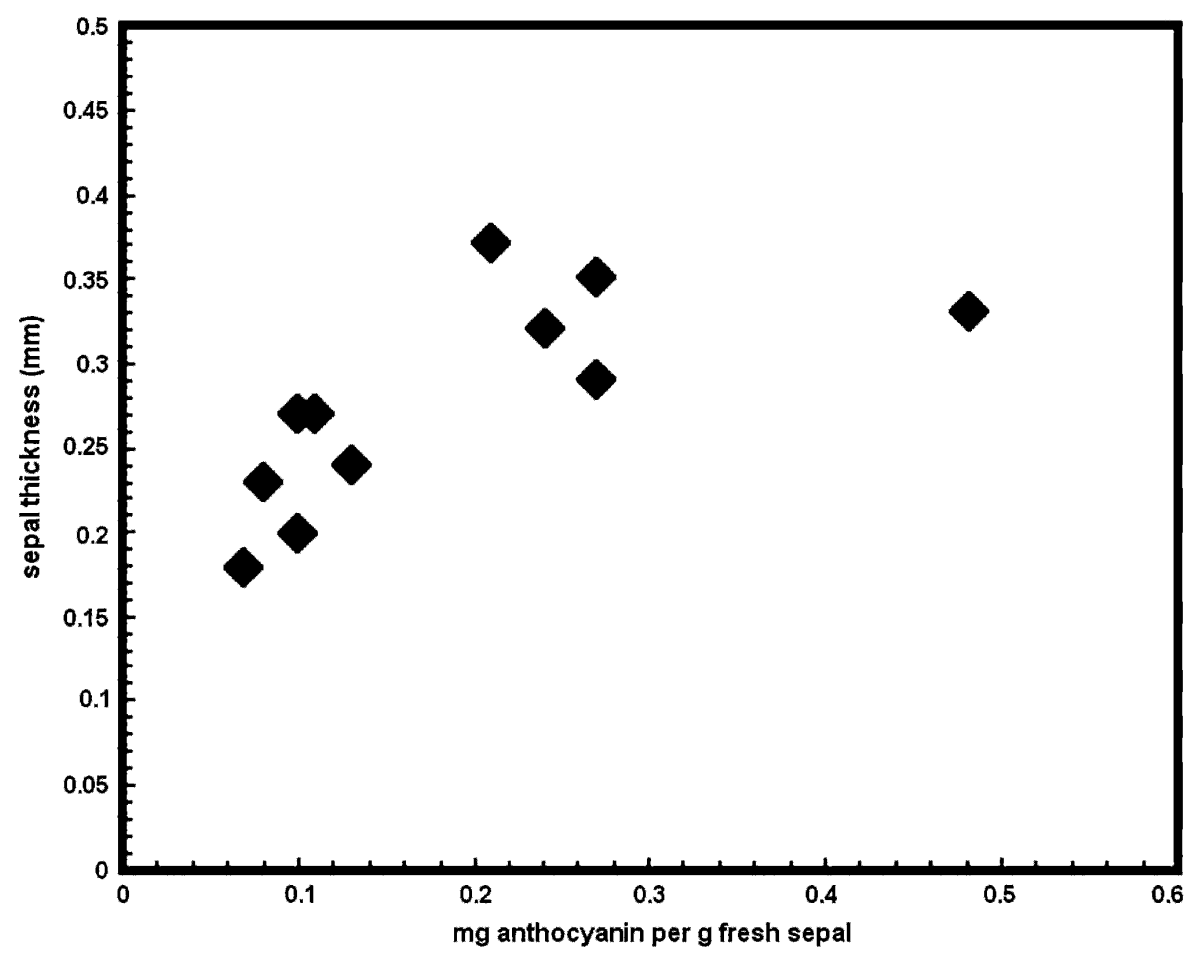

Fig. 3. Average sepal thicknesses for several hydrangea cultivars as a function of the average extractable anthocyanin concentration (mg of delphinidin-o-glucoside per $\mathrm{g}$ of fresh sepal) for that cultivar.

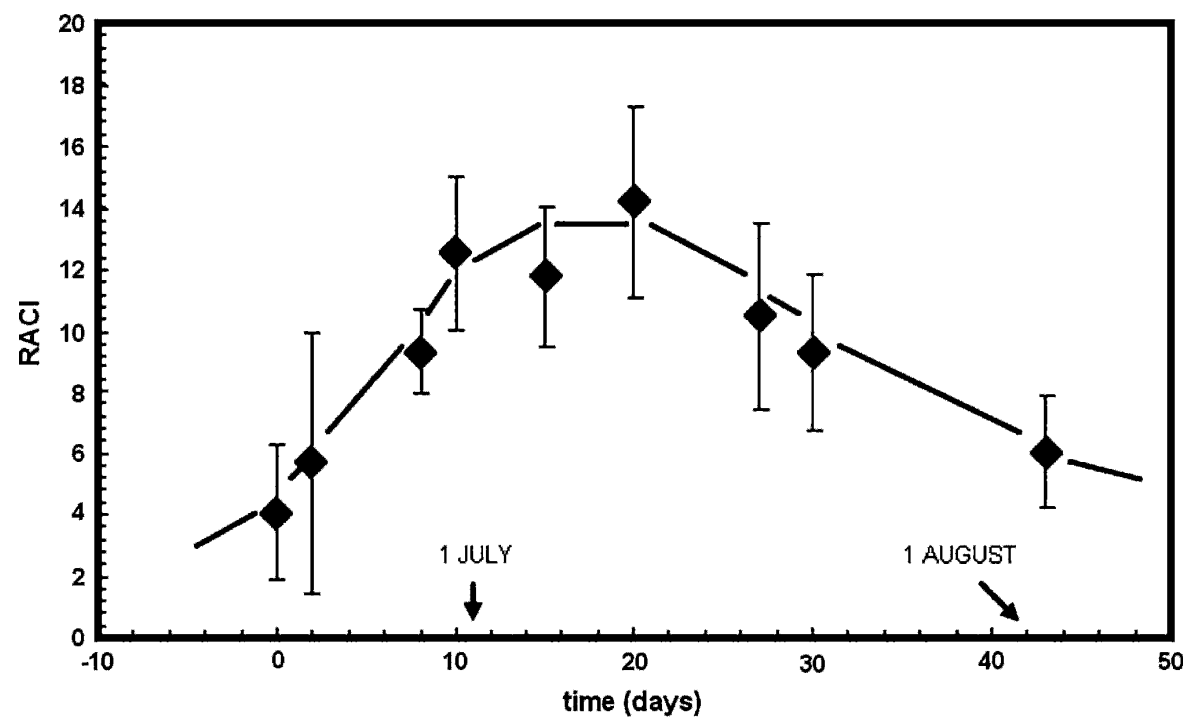

Fig. 4. Red anthocyanin content index (RACI) measured with the field-portable meter for the red sepals of a 'Masja' inflorescence as a function of bloom time. Measurements were taken during Summer 2006 in the gardens of BackCountry Research.

a few weeks elapsed between the initial bud expression and this time. The RACI can also be expressed in terms of $\mathrm{mg}$ of anthocyanin per $g$ of fresh sepal upon use of the calibration provided in Fig. 1. Figure 4 also illustrates the variation (error bars) in sepal-to-sepal coloration within a single inflorescence as well as the length of time that the shrub is at maximum coloration. preserving the color of hydrangea could be easily investigated to extend the hydrangea's vase life as a cut flower.

In summary, the calibrated field-portable meters will allow the direct measurement of anthocyanin concentration in both red and blue hydrangea sepals. Quantitative determinations of sepal color as a function of time (color history) in the field will be readily measurable as a function of environmental variables. In conjunction with other measurements, such as $\mathrm{pH}$ and aluminum content that can then be made on the same sepals, these meters will be invaluable in ascertaining the chemical mechanism for this shrub's unique colorations.

\section{Literature Cited}

Asen, S., H.W. Siegelman, and N.W. Stuart. 1957. Anthocyanin and other phenolic compounds in red and blue sepals of Hydrangea macrophylla var. Merveille. Proc. Amer. Soc. Hort. Sci. 69:561-569.

Asen, S., N.W. Stuart, and A.W. Specht. 1960. Color of Hydrangea macrophylla sepals as influenced by the carry-over effects from summer applications of nitrogen, phosphorous, and potassium. Proc. Amer. Soc. Hort. Sci. 76: 631-636.

Giusti, M.M. and R.E. Wrolstad. 2000. Characterization and measurement of anthocyanins by UV-visible spectroscopy, Unit F1.2. In: Current protocols in food analytical chemistry. Wiley, New York. 12 pp.

Kondo, T., Y. Toyama-Kato, and K. Yoshida. 2005. Essential structure of co-pigment for blue sepal-color development of hydrangea. Tetrahedron Lett. 46:6645-6649.

Moncada, M.C., S. Moura, M.J. Melo, A. Roque, C. Lodeiro, and F. Pina. 2003. Complexation of aluminum(III) by anthocyanins and synthetic flavylium salts: a source for blue and purple color. Inorg. Chim. Acta 356:51-61.

Pharr, K.E., K.M. Mayhew, and H.D. Schreiber. 2006. Anthocyanin content in hydrangea sepals. J. Undergrad. Chem. Res. 5:49-53.

Robinson, G.M. 1939. Notes on variable colors of flower petals. J. Amer. Chem. Soc. 61:16061607.

Takeda, K., M. Kariuda, and H. Itoi. 1985. Blueing of sepal color of Hydrangea macrophylla. Phytochemistry 24:2251-2254.

Toyama-Kato, Y., K. Yoshida, E. Fujimori, H. Haraguchi, Y. Shimizu, and T. Kondo. 2003. Analysis of metal elements of hydrangea sepals at various growing stages by ICP-AES. Biochem. Eng. J. 14:237-241.

van den Berg, A.K. and T.D. Perkins. 2004 Evaluation of a portable chlorophyll meter to estimate chlorophyll and nitrogen contents in sugar maple (Acer saccharum Marsh.) leaves. For. Ecol. Mgt. 200:113-117.

van den Berg, A.K. and T.D. Perkins. 2005. Nondestructive estimation of anthocyanin content in autumn maple leaves. HortScience 40:685-686.

Yoshida, K., Y. Toyama-Kato, K. Kameda, and T. Kondo. 2003. Sepal color variation of Hydrangea macrophylla and vacuolar $\mathrm{pH}$ measured with a proton-selective microelectrode. Plant Cell Physiol. 44:262-268. 